\title{
F(750), We Miss You as a Bound State of 6 Top and 6 Antitop Quarks, Multiple Point Principle
}

\author{
Holger F. Bech Nielsen*† \\ Niels Bohr Institute, Blegdamvej 15-21, DK 2100 Copenhagen Ø, Denmark \\ D.L. Bennett \\ Brooks Institute, Copenhagen, Denmark \\ C.R. Das ${ }^{\ddagger}$ \\ BLTP, JINR, Dubna, Moscow, Russia \\ C.D. Froggatt ${ }^{\S}$ \\ Glasgow University, Glasgow, $U K$ \\ L.V. Laperashvili đ \\ ITEP, National Research Center "Kurchatov Institute”, Moscow, Russia
}

\begin{abstract}
We review our speculation, that in the pure Standard Model the exchange of Higgses, including also the ones "eaten by $W^{ \pm}$and Z", and of gluons together make a bound state of 6 top plus 6 anti top quarks bind so strongly that its mass gets down to about $1 / 3$ of the mass of the collective mass $12 m_{t}$ of the 12 constituent quarks. The true importance of this speculated bound state is that it makes it possible to uphold, even inside the Standard Mode, our proposal for what is really a new law of nature saying that there are several phases of empty space, vacua, all having very small energy densities (of the order of the present energy density in the universe). The reason suggested for believing in this new law called the "Multiple (Criticality) Point Principle" is, that estimating the mass of the speculated bound state using the "Multiple Point Principle" leads to two consistent mass-values; and they even agree with a crude bag-model like estimate of the mass of this bound state. Very unfortunately the statistical fluctuation so popular last year when interpreted as the digamma resonance $F(750)$ turned out not to be a real resonance, because our estimated bound state mass is just around the mass of $750 \mathrm{GeV}$.
\end{abstract}

Corfu Summer Institute 2016 "School and Workshops on Elementary Particle Physics and Gravity" 31 August - 23 September, 2016

Corfu, Greece

\footnotetext{
*Speaker.

†hbech@nbi.dk, hbechnbi@gmail.com

łcrdas.crdas@gmail.com, crdas@cftp.ist.utl.pt, das@theor.jinr.ru

$\S$ c.froggatt@physics.gla.ac.uk

『laper@itep.ru
} 


\section{Introduction}

Main ideas The main point of the present contribution is to attempt to make the listeners believe in our proposal for a "new law of nature" - which we shall call the "Multiple (criticality) Point Principle" $[1,2,3,4,5,6,7,8,9,10,11,12,13,14,15,16,17,18,19$, 20], shortened to MPP - which delivers some information about the coupling constants (or better the parameters of the theory, say e.g. the Standard Model). In realizing the picture, which we suggest in connection with this new law of nature, we further need to have that there exists an extremely strongly bound state[21, 22, 23, 24, 25, 26, 27, 28, 29, 30, 31, 32, 33, 34, 35, 36] of six top plus six antitop quarks, and thus our main points are:

\section{- Strongly Bound State}

We speculate that mainly due to exchange of Higgs bosons a system of 6 top plus 6 anti top quarks bind so strongly as to make a bound state with appreciably lower mass than that of 12 separate quarks and anti quarks.

\section{- Multiple Point Principle}

We propose a new law of nature (MPP) saying, that - somewhat mysteriously may be - the coupling constants and other parameters, such as the Higgs mass squared, get adjusted so as to guarantee, that there are several vacua all with very small energy densities (=cosmological constants).

By a strange accident there appeared around Christmas 2015 a peak in the spectrum, now known to be statistical fluctuation, but at that time looking like a resonance produced in LHC and decaying into pairs of photons with a mass of $750 \mathrm{GeV}[37,38,39,40]$ - thus called a digamma $\mathrm{F}(750)$-. This was a strange accident, because it is the main point of the present article, that we calculate the mass of the bound state of the 6 top +6 anti tops in our scheme - speculation inside pure Standard Model - in three different ways using our "law" MPP, and obtain within our very low accuracy something perfectly consistent with the mass being $750 \mathrm{GeV}$ ! We therefore really miss this by now no longer statistically viable digamma state.

An extremely tiny hope may though today be represented by the fact that a new presumably again purely statistical fluctuation at LHC corresponds to producing a resonance with mass 650 $\mathrm{GeV}$ decaying into two Z-bosons. The experimental mass determinations are so that $650 \mathrm{GeV}$ is quite distinguishable from $750 \mathrm{GeV}$, but our theoretical accuracy in predicting the mass of our bound state is not sufficient to distinguish $650 \mathrm{GeV}$ from $750 \mathrm{GeV}$, and so for us the new $650 \mathrm{GeV}$ fluctuation would be fine. According to our estimates [41] the easiness of observing the decay of the $6 \mathrm{t}+6 \bar{t}$ bound state decaying into $\gamma \gamma$ and into $Z Z$ are rather similar, although the dominant decay would be into $t \bar{t}$, but the latter may be more difficult "see".

\section{Multiple Point Principle (MPP)}

Our proposal for a new law of nature - Multiple Point Principle (MPP) - (first by Don Bennett and myself) means that there shall exist several vacua with very small energy density.

Three Vacua in Standard Model: For simplicity and trustability we in this talk restrict ourselves to the pure Standard Model and only the following three vacua: 


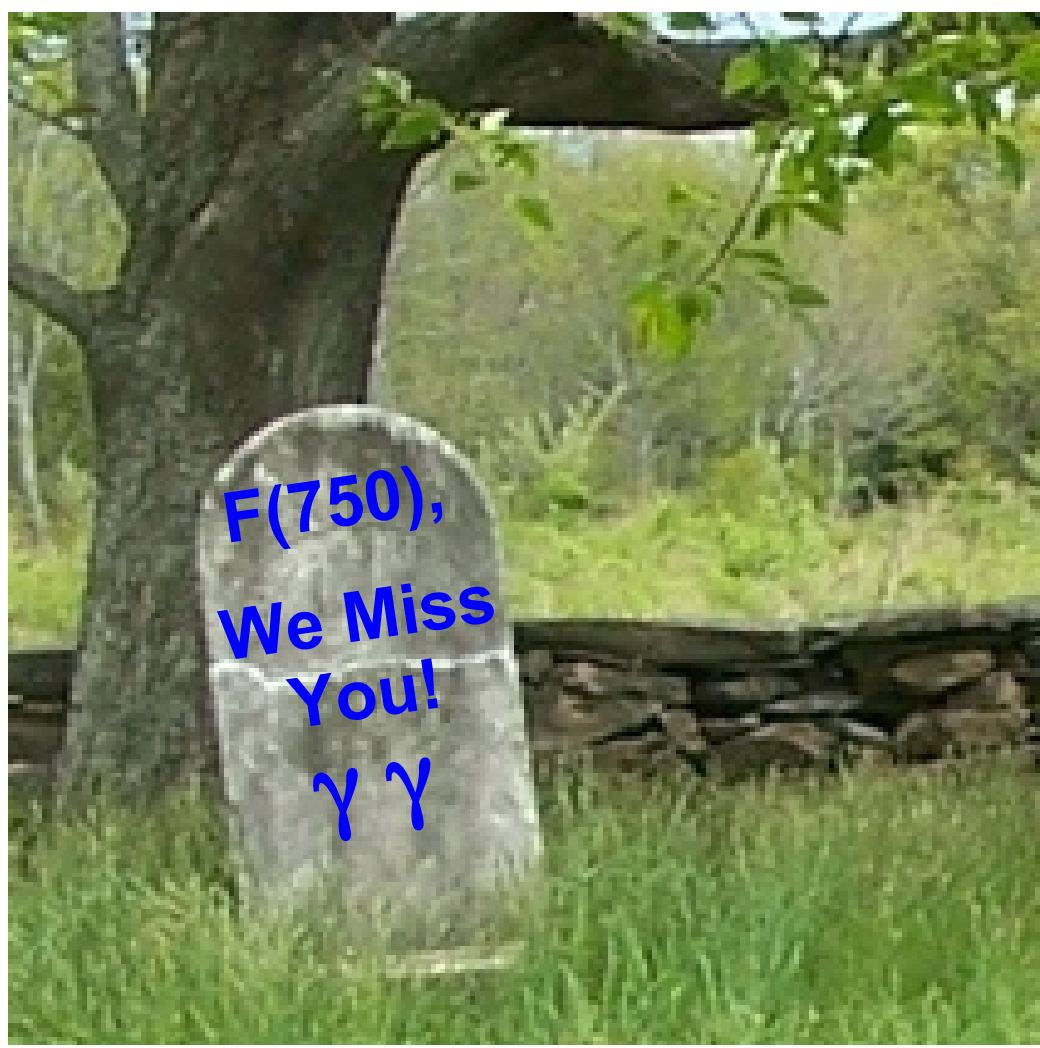

Figure 1: We miss you!

- Present: The vacuum, in which we live, in the sense that, if we in practice find a place with zero density of material, then that region is in the state of the "present vacuum".

- High (Higgs) field vacuum: This vacuum is a state, in which the Higgs field is at a minimum in the Higgs-effective potential $V_{\text {eff }}\left(\phi_{H}\right)$ having a value of the Higgs field near $\phi_{H} \sim 10^{18}$ $\mathrm{GeV}$. It is known that, in the pure Standard Model, it seems that the energy density of this vacuum is slightly negative (with 3 standard deviations from being just zero).

- Condensate vacuum: This third vacuum is a very speculative possible state inside the pure Standard Model, which contains a lot of strongly bound states distributed smoothly in space, each bound from 6 top +6 anti top quarks.

\section{Can use Multiple Point Principle together with Any Model (side-remark)!}

In the present talk I shall concentrate on the version "several vacua, that all have very small energy densities" i.e. MPP.

But an older version had it: "Several vacua all have the same energy density (with some accuracy, that can be discussed)" in MPP.

Also one does not need to assume just the Standard Model as I shall do in the present talk. For instance Roman Nevzorov with some of us (Froggatt and me) [12, 13, 14, 42, 43, 44, 45, 46, 47, $48,49,50,51,52,53,54,55,56,57,58,59]$. assumed a supersymmetry only broken tinily in one vacuum, but much more strongly broken in e.g. the present vacuum. 

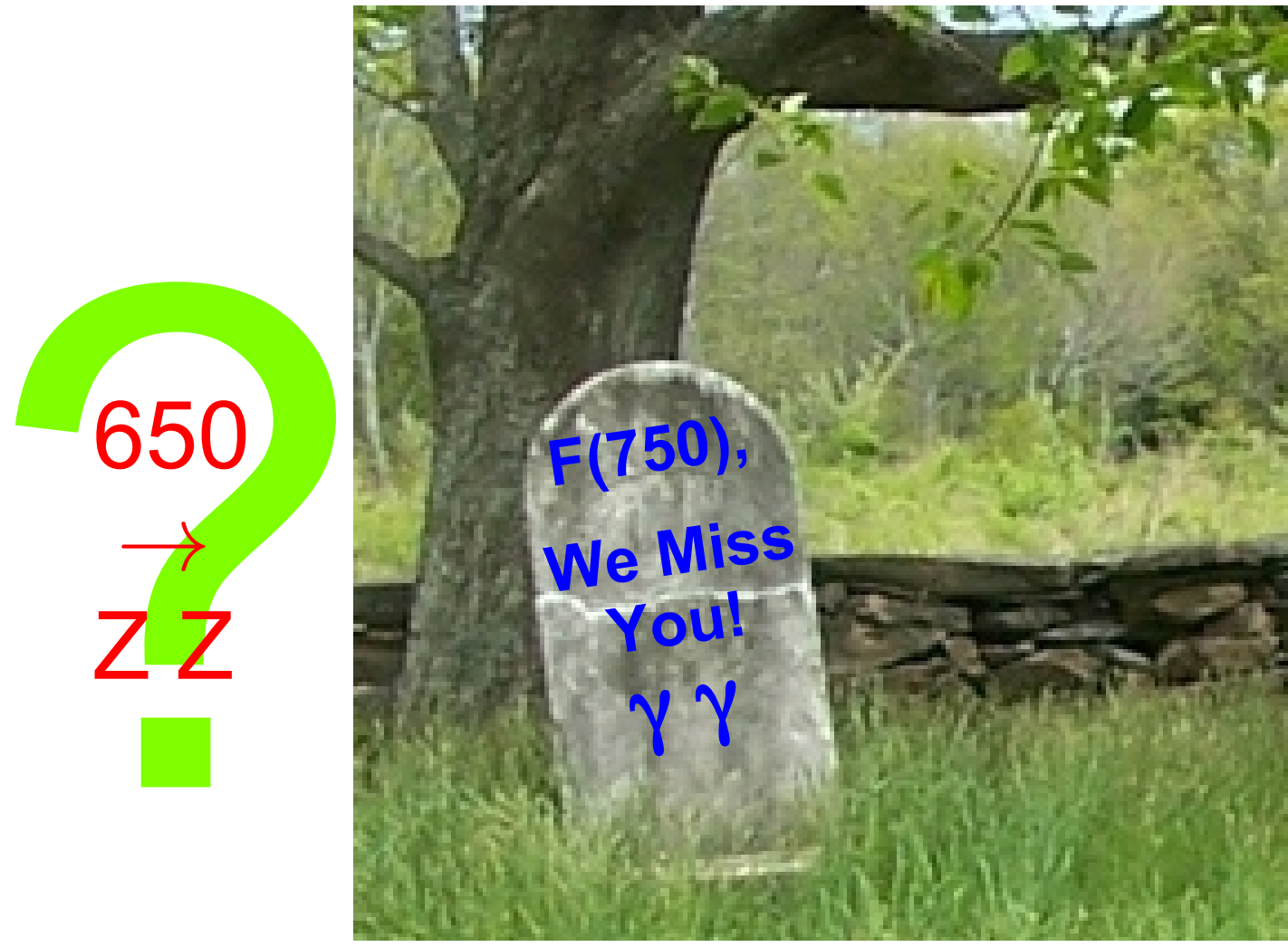

Figure 2: We miss you! Must have a reason!

The works with Roman Nevzorov extend the Standard Model with Susy etc. [12, 13, 14, 42, $43,44,45,46,47,48,49,50,51,52,53,54,55,56,57,58,59]$. (Otherwise in the present talk I only keep the Standard Model.)

Assuming some of the vacua to have only tinily broken susy, a tiny cosmological constant in the almost susy unbroken vacuum could be transferred - by means of a very accurate MPP to the present vacuum, and a rather successful fitting/derivation of the astronomically determined cosmological constant could be achieved! (An extra five-plet could make the fitting extremely good.)

Also the original idea on the basis of which we - Don Bennett and I - invented the "multiple point principle" was based on a model called AntiGUT[60, 61, 62, 63], which extends the Standard Model. However it has first new physics rather close to the Planck scale, actually in it each family of fermions has its own system of gauge groups. The gauge bosons are also in families!

\section{Finetuning of Parameters, Couplings:}

Our "multiple point principle" is really just an assumption about the coupling constants - in the Standard Model, if we as in this talk take the model to be pure Standard Model - being finetuned so as to make the three vacua proposed have just zero energy density $V_{\text {present }}, V_{\text {condensate }}, V_{\text {high field }}=0$ (with say the accuracy of the order of the astronomically found energy density in the "present vacuum" $\sim 75 \%$ of the total energy density in the present universe).

I.e. MPP provides 3 restrictions between the parameters of the model in question, here the Standard Model, from which the $\sim$ zero energy densities in all three vacua follows. 
Multiple Point Principle means Relations between the couplings and other parameters:

$$
\begin{aligned}
V_{\text {present }}\left(\Lambda_{C C}, g_{t}, m_{H}^{2}, \Lambda_{Q C D}, \ldots\right) & =0 \\
V_{\text {condensate }}\left(\Lambda_{C C}, g_{t}, m_{H}^{2}, \Lambda_{Q C D}, \ldots\right) & =0 \\
V_{\text {high field }}\left(\Lambda_{C C}, g_{t}, m_{H}^{2}, \Lambda_{Q C D}, \ldots\right) & =0
\end{aligned}
$$

Here we wrote explicitly the following parameters of the Standard Model:

$$
\begin{aligned}
\Lambda_{C C} & : \text { The cosmological constant } \\
g_{t} & : \text { The top Yukawa coupling } \\
m_{H}^{2} & : \text { Higgs mass squared } \\
\Lambda_{Q C D} & : \text { The scale parameter of QCD }
\end{aligned}
$$

Whether these parameters are renormalized or bare does not matter so much here.

$V_{\text {present }}, V_{\text {cindensate }}, V_{\text {high field }}$ are the vacuum energy densities for the three speculated vacua.

\section{Use of Multiple Point Principle:}

Taking the experimental values for all the Standard Model parameters except for say $\Lambda_{C C}, m_{H}^{2}$ and $g_{t}$, we could look at it such that e.g. $V_{\text {present }}=0$ fixes the cosmological constant $\Lambda_{C C}$ to essentially zero. (It is very small indeed.) Then $V_{\text {high field }}=0$ (meaning the energy density of the vacuum having the very high Higgs field $\phi_{H} \approx 10^{18} \mathrm{GeV}$ ) could be taken to predict the Higgs mass, and the $V_{\text {condensate }}=0$ to predict, say, the $g_{t}$ Yukawa coupling. In fact Colin Froggatt and H.B.Nielsen PREdicted the Higgs mass many years ago[5] to be $135 \mathrm{GeV} \pm 10 \mathrm{GeV}$ from such an MPP-assumption.

In fact the speaker (H.B.N.) was portrayed long before the Higgs was found experimentally at LHC together with Mogens Lykketoft - a previous member of the Danish cabinet - with the number $135 \mathrm{GeV} \pm 10 \mathrm{GeV}$ written partly behind the head of this member of the cabinet, meaning our PREdiction for the Higgs boson mass. Since the much later determined experimental mass turned out to be $125.06 \mathrm{GeV} \pm .21 \mathrm{GeV}$ (statistical uncertainty) \pm 0.11 (systematic uncertainty), we had agreement for our PREdiction deviating only by one standard deviation (of our theoretical uncertainty in the old time in the 90s):

Having in mind that the "present vacuum" as well as the "high (Higgs) field vacuum" basically correspond to two different minima in the effective potential $V_{e f f}\left(\phi_{H}\right)$ for the Higgs field $\phi_{H}(x)$, we see that the statement of these two vacua having the same energy density means, that there is in the effective potential $V_{e f f}\left(\phi_{H}\right)$ as a function of the Higgs field strength $\phi_{H}$ two equally deep minima. Our PREdiction consisted in fitting the Higgs mass $m_{H}$ to arrange the effective potential for the Higgs field $V_{\text {eff }}\left(\phi_{H}\right)$ to have such two minima of equal depth.

On the figure 4 the curve touching the abscissa axis in two points is the one corresponding to the Higgs mass as predicted from the "Multiple Point Principle", while the curve crossing the abscissa a couple of times and only touching it at the "present vacuum" Higgs field of the wellknown $246 \mathrm{GeV}$ corresponds to a somewhat lower value of the Higgs mass. With the modern top mass value and the very accurate theoretical calculations performed in [64, 65], the Higgs mass predicted from the "multiple point principle" would be $129.4 \mathrm{GeV}$ rather than the old estimate 


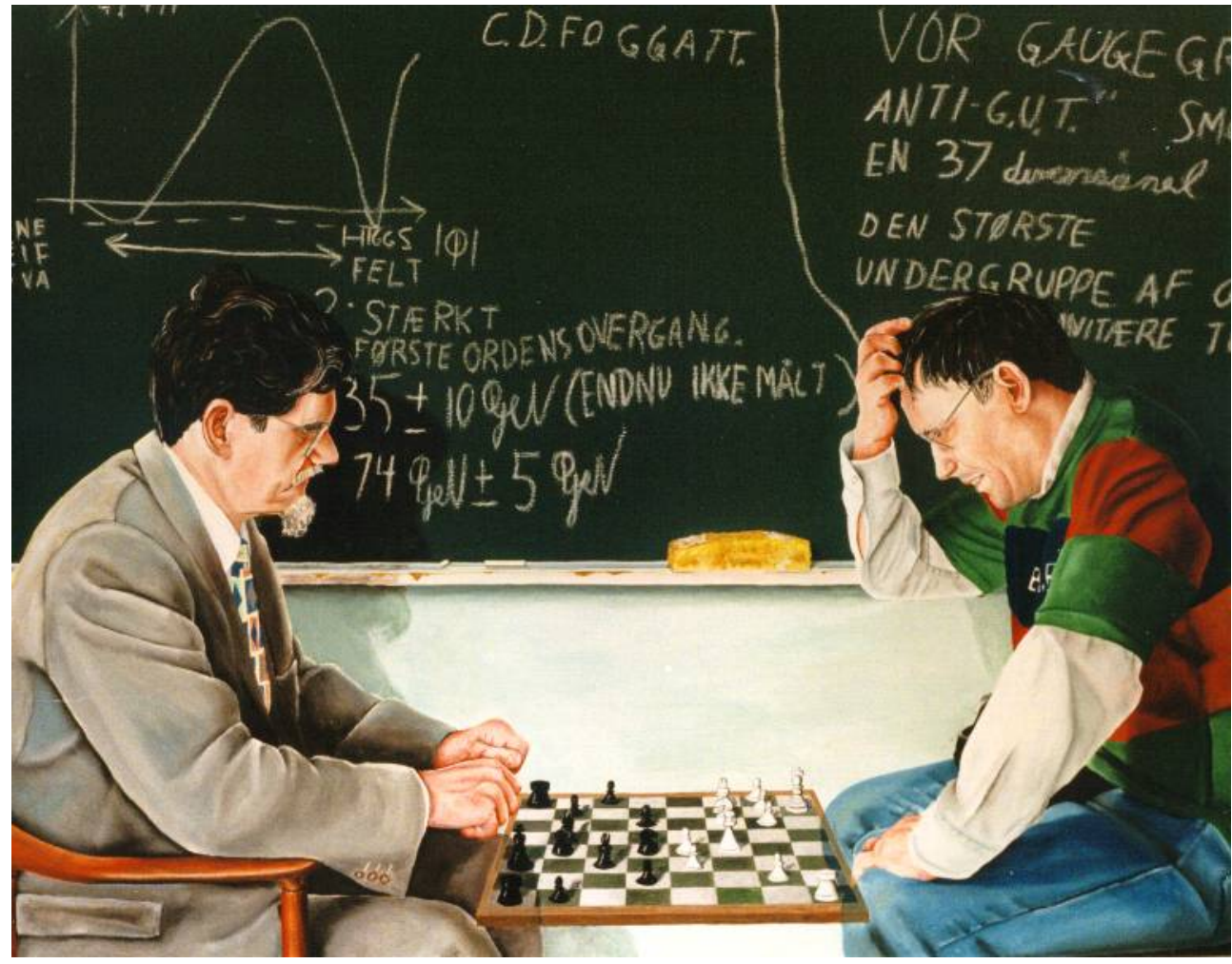

Figure 3: Chess!

$135 \mathrm{GeV}$. The uncertainty in the top-quark mass measurement is indeed very important and it might be better to look at the "multiple point principle"-prediction as giving a relation between the top and the Higgs masses. In any case: while in the old accuracy of calculation we got only one standard deviation from the by now measured Higgs mass, the present deviation is rather 3 standard deviations because of the increased top-quark mass measurement accuracy, in spite of that the predicted Higgs mass has indeed moved in the good direction from the $135 \mathrm{GeV}$ towards 129.4 $\mathrm{GeV}$; but the decrease in the uncertainty has been even faster.

It should also be mentioned that Colin Froggatt, Yasutaka Takanishi and the speaker [66] proposed the alternative idea of rather than quite degenerate vacua (having same energy density) to require just META-stability, which should be expected as what should be important in as far as that could influence whether our vacuum - the present vacuum - decays to the high Higgs field or not. This meta-stability - also long before the Higgs was found experimentally at LHC - PREdited a mass of the Higgs being $121 \mathrm{GeV}$. Actually this was even a bit closer to the actual Higgs mass than our old $135 \mathrm{GeV}$. But the deviation of the meta-stability PREdiction is to the opposite side: Relative to "META-multiple point principle" the experimental Higgs mass $125 \mathrm{GeV}$ is slightly to the high side of the PREdicted one.

\section{A Remarkable Side-Point:}

The second minimum in the Higgs effective potential corresponding to what, we call the "High field" vacuum, has an expectation value for the Higgs field $\phi_{H}$, which is remarkably close (order of magnitudewise) to the Planck energy scale! This should not be an accident, but rather explained: 


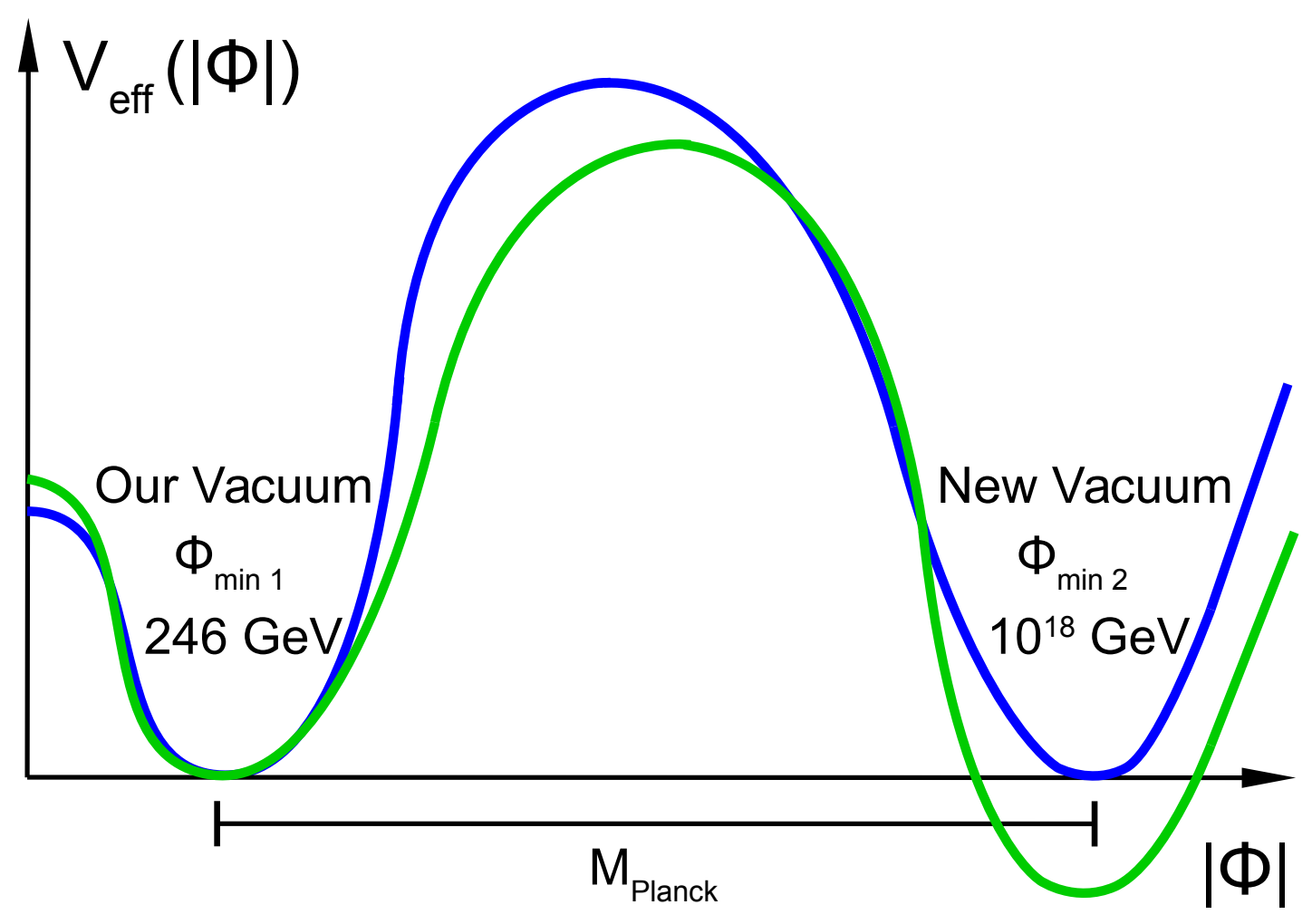

Figure 4: Two different minima in the effective potential for the Higgs field.

the Planck energy scale is the "fundamental physics scale" for both energy and Higgs fields; they have the same dimension.

Two of the vacua, which we discuss today have for some reason exceptionally small, say, Higgs field, while the "high field" vacuum has the "normal" order of unity in Planck units value for its Higgs expectation value. So we rather ask the question:

Why do the two vacua, "present vacuum" and "condensate vacuum", not have Planck scale, say, Higgs fields? (Let me for the moment postpone a discussion of the fact that indeed we have an explanation[26, 27] from the "multiple point principle", that these two vacua have exceptionally small Higgs expectation values scale.)

\section{Reason}

But Why should we believe in the postulate of the Multiple Point Principle ?

\section{- Need Coupling Explanations:}

It is clear that there are some parameters in the Standard Model that take so special values, that it cries for an explanation; there are fine tuning problems:

- Even with great effort e.g. Graham Ross[67] could not get the factor $\Delta$ with the inverse of which the Higgs is too light further down than about 1/20. 
- Cosmological constant? Why is it so small?

- Derivations: In models which allow somehow influence from the future[68] to adjust coupling constants one may make some "derivations" of MPP.

- Empirical: But really it is the main point of today's talk to deliver some empirical support (our PREdiction of the Higgs mass, and two derivations of the same bound state mass)

\section{Theoretical Reason: "Plural of Cosmological Constant"}

1 We have to assume that the energy density in the "present vacuum" is very small compared say to the Planck energy[69] density, because it has been well-known since long before the measurements with supernovae A1 settled it to be non-zero.

2 This assumption does not become essentially less beautiful or more complicated by "putting it in plural": Several vacua have very small energy density/ cosmological constant compared to say the Planck energy density or the Higgs energy density or compared to most high energy physics contributions.

(This argument came from private conversation with L. Susskind.)

But each time you fix the energy of one more vacuum energy density you get one more relation between the parameters/ couplings of the theory.

It may even be more beautiful to talk about several/all vacua than just a special one that must be specified.

\section{Theoretical Reason: Extremizing Something, Positive Energy}

A. Assume that energy-density should be positive or zero. i.e. the bottom in the Hamiltonian density is at least zero. This will restrict the coupling constants and other parameters - e.g. Higgs mass - to some polyhedron-like figure with curved sides, where the sides correspond to one possible vacuum or the other having just zero energy density.

B. Assume that the couplings and parameters inside the positivity restriction are selected by minimizing something / some function of these couplings and parameters (a generic or "random" function).

Using the anthropic principle[70] the function could suggestively be the number of human beings in the universe resulting with the couplings etc. in the point in parameter-space considered.

On the figure 5 I have drawn the contour curves - really contour surfaces - for the function to be minimized.

\section{Very often Minimum of Function occurs in Corners}

The crucial point is, that - especially in a high dimensional coupling-constant and parameter space - the minimum will very often fall in a corner (where several sides cross) of the polyhedronlike region with curved sides (violet).

But the "sides" correspond to different vacua having zero energy density. So a corner corresponds to several vacua all having zero energy density $\rightarrow$ "Multiple Point Principle"! 


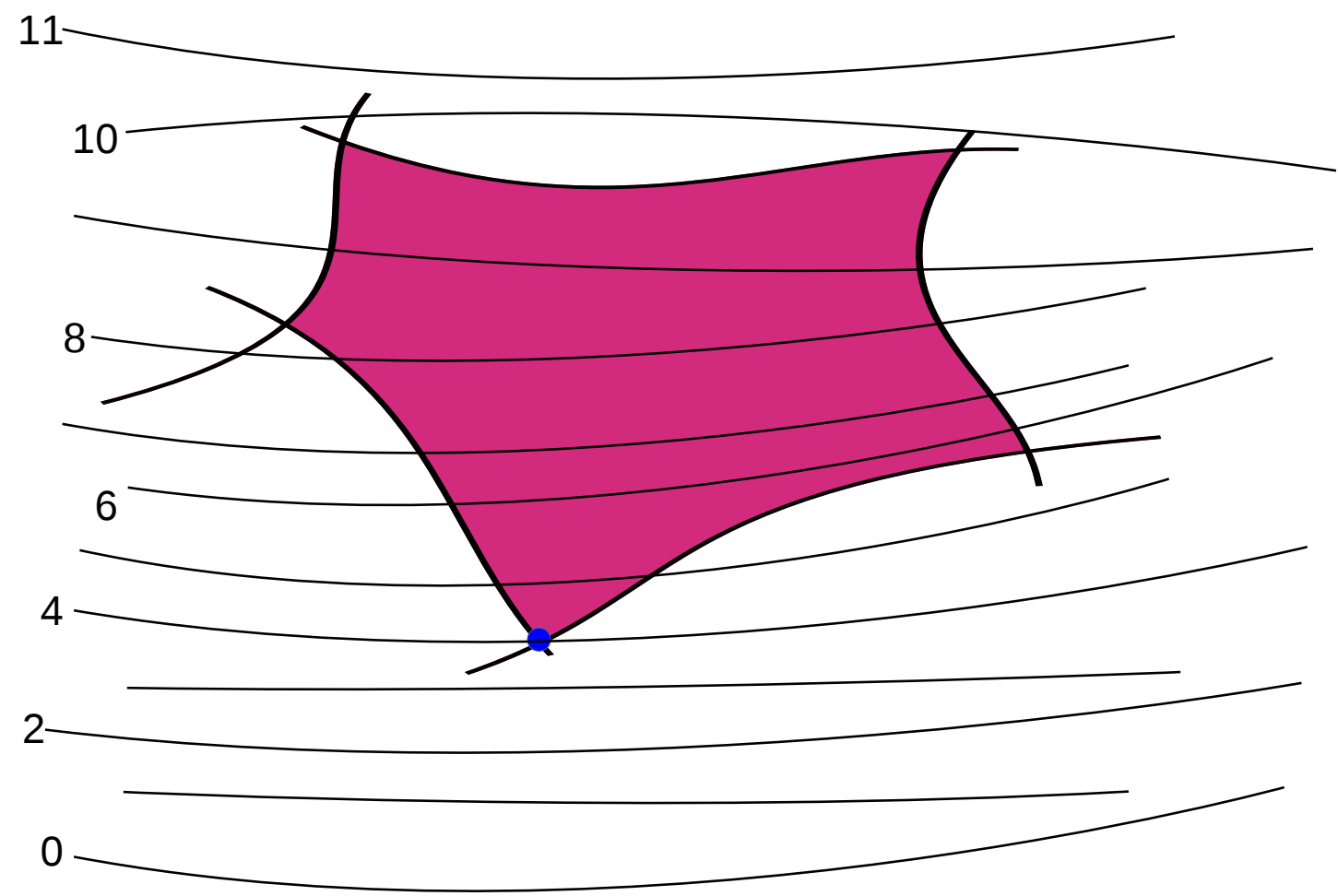

Figure 5: Contour curves.

\section{Theoretical Reason; Our Bennett's and Mine Original Explanation [3, 4, 5, 6]}

One assumes, that some extensive quantities / commodities i.e. some integrals over space time of say fields raised to some powers etc. - say Higgs field squared - are fixed by "God"/ some law, rather than as I think we would usually think, it is the couplings themselves that are selected by "God".

To really obtain the Multiple Point Principle as we wanted you must fix some integrals over the four dimensional space time to "God given values". Mathematically, however, what we did was very analogous to what one does for a three dimensional system in working with micro-canonical ensembles, when one e.g. fixes the energy, the volume, and the number of moles of say water. Then without specifying these extensive quantities very accurately, one can get that the intensive quantities temperature and pressure gets fine tuned to the triple point, see figure 6 . In the bottle with water, ice and vapour one has actually fixed the amount of mols of water molecules, the volume and the total energy of the system inside the bottle. That is to say extensive quantities were fixed, but not to any very special values. The pressure and temperature, however, come out with the very special triple point values. The name for our principle is derived in analogy with this notation "triple point" for the (pressure, temperature) combination appearing at such a meeting of several (here three) phases.

One should take this analogy of the method for deriving the Multiple Point Principle with the often found slush having to have a fixed temperature as very encouraging! When nature naturally provides slush and just $0^{0}$ Celsius for us, it might also make the MPP-situation for the couplings. 


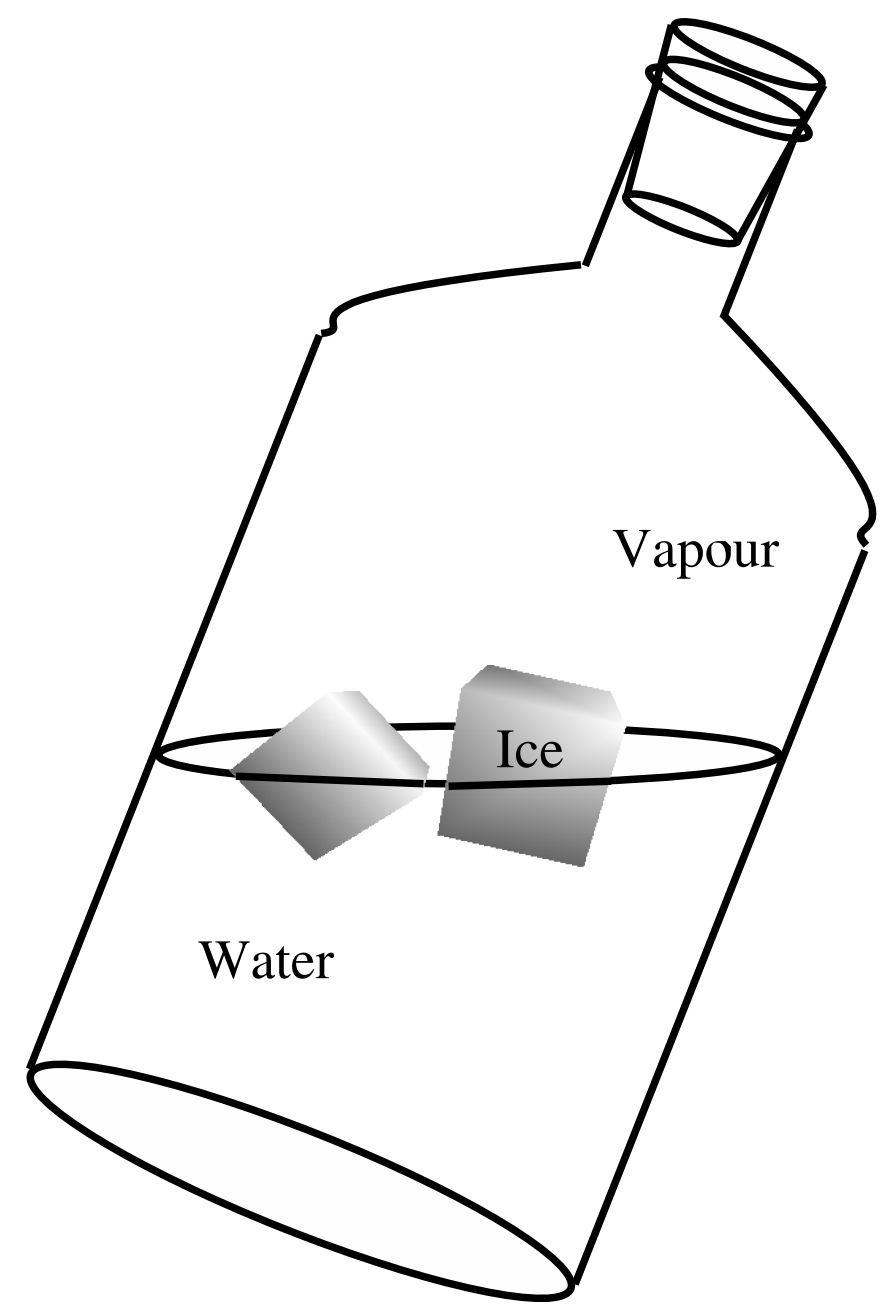

Figure 6: Vapour, water and ice equilibrium in a bottle.

\subsection{Model Reasons}

It should be stressed that in specific model-pictures one can also derive the "Multiple (Criticality) Point Principle" supposedly though it is only possible in models allowing the coupling constants to depend on the future too. Ninomiya and one of us (HBN) "derived" it in the imaginary action type of theory, and somewhat similarly in a nonlocal theory by Stillits [71, 72] and it was done in babyuniverse theory, see works by Kawana et al. [15, 16, 17, 18].

\section{The Difficulty of the Bound State}

When we - as we now do - want to check if the "multiple point principle" is a true/valid law of nature, we have the difficulty that an important role is played by a bound state with which the vacuum, which we call "condensate vacuum", is filled.

Fundamentally one cannot calculate completely perturbatively, when one calculates on a bound state! 
Especially when, as we expect, the bound state is very strongly bound, the calculation will involve a non-perturbative calculation, while in quantum field theory the only truly working method is perturbation theory. So we are advised to make doubtful approximations using less reliable techniques such as bag models or mean field theories, or we have to be extremely lucky in that something can be actually calculated accurately. Really one should in the long run make some very hard numerical calculations by computer, but so far we have ourselves only made very poor computations by hand waving approximations.

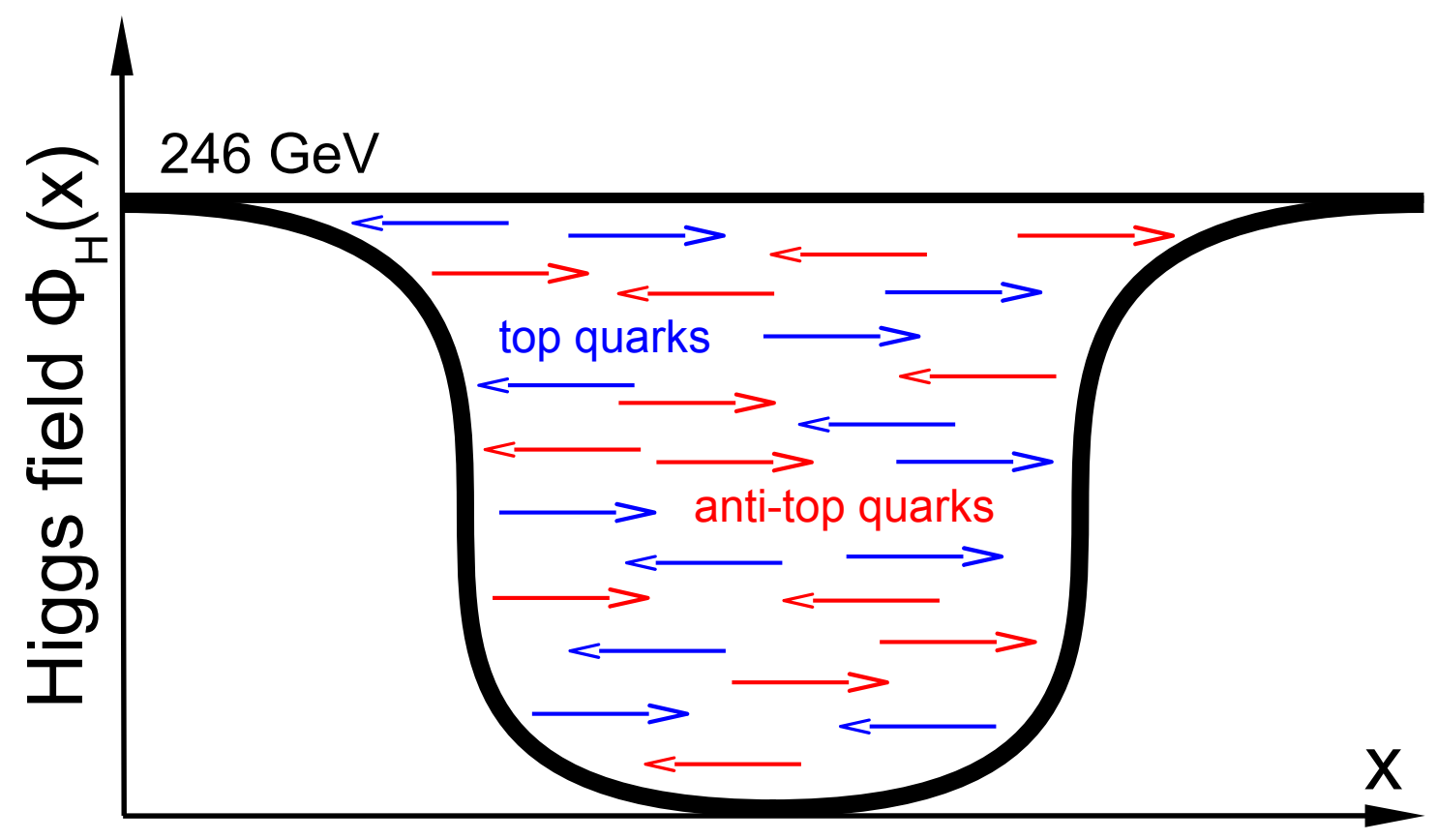

Figure 7: Bag in Higgs field filled with top and anti-tops.

\section{Lucky Overdetermined Situation}

Luckily we are with the "multiple point principle"(to be tested) in the very good situation calculationally, that in addition to knowing already from experiment all the parameters of the Standard Model today, we have the three extra equations, if MPP is assumed. So we can use this information to help us through the caculational problems with the bound state.

\section{Checking MPP by Calculating Mass of the Bound State in Several Ways}

Since the calculation is non-perturbative and thus difficult so that we can only very crudely compute say the mass of the bound state, it is suggestive to take it as a parameter. Then we may formulate testing the "multiple point principle" as evaluating, by different assumptions inside MPP, the mass of the bound state several times and see if we shall get the same mass each time we calculate (by using different information from MPP).

\section{Really Check Bound State Mass Obtained from Degeneracy of Vacuum Pairs}


Our technique - today - is to estimate/calculate the value of the mass of the bound state of 6 top and 6 anti top quarks speculated to exist in our picture/model. (Since we have two relative energy density predictions - ignoring the absolute smallness of the cosmological constant - we get two such bound state mass fits.) In addition we can seek to obtain the bound state mass by building a bag-model-like ansatz for the bound state and estimates its mass. Thus we get, by using our multiple point principle, two a priori different mass predictions for the bound state, in addition to the bag model estimate of the mass as being that of a bound state.

\section{Our Three Bound State Mass Fits:}

- High field fit: We fit to get a tiny correction to the Higgs mass relative to the running selfcoupling so as to ensure the MPP - requirement that the "present vacuum" be degenerate with the "high field vacuum": Fitting mass $m_{\text {from high field fit }} \approx 700 \mathrm{GeV}$ to $800 \mathrm{GeV}$.

- Condensate vacuum fit: We fit the mass to be such that the binding between the bound states in a region filled with such particles in the lowest energy density state just gets zero/same energy density as in the present vacuum. With a simple but accidentally almost true assumption, discussed in section 7 below, we fit the mass to $m_{\text {from condensate fit }} \approx 4 m_{t}=692 \mathrm{GeV} \pm$ $100 \mathrm{GeV}$, say.

- Ansatz calculation: We make a bag-model- like crude ansatz for the bound state of the 6 top +6 anti top quarks and seek the minimum energy/mass by varying bag radius $R$. With very crude inclusion of various corrections, we reach the mass estimate $m_{\text {bag-model }} \approx 5 m_{t}=865$ $\mathrm{GeV} \pm 200 \mathrm{GeV}$, say.

\section{Plan}

\section{Introductionary sections:}

- Heading: F(750), We miss you!

- Introduction: New Natural Law of Nature, and Bound State.

- MPP: The New law, "Multiple Point (Criticality) Principle".

- Bound Bound state of 6 top and 6 anti top quarks.

- Reasons: Attempts to explain why MPP.

- Plan: Plan of the talk.

\section{Three Calculations:}

- High: The mass of the bound state that could arrange the stability of our vacuum to be just borderline stable w.r.t. the Higgs field.

- Condensate: The mass of the bound state making the "condensate vacuum" degenerate in energy density to the "present vacuum". 
- Bag: Bag model estimation of the mass of the bound state.

\section{Important Application plus Conclusion:}

- Hierachy: Our multiple point principle enforces the weak scale of energy compared to the one of the high higgs field $\sim$ Planck scale.

- Conclusion: Telling that you should now believe our MPP law of nature!

\section{High}

\section{Small Correction by Laperashvili, Das, and me to "High field Vacuum" Energy Density [35]}

From De Grassi et al.'s calculation[64] of the effective Higgs field potential $V_{e f f}\left(\phi_{H}\right)$ there is a high scale minimum in this potential, but it goes slightly under 0 so that the present vacuum is unstable for the experimental Higgs mass 125.09 \pm 0.24 , while the value that would have made the second minimum just degenerate with the present vacuum energy density would be rather $\left.m_{H}\right|_{\text {from MPP De Grassi... }}=129.4 \mathrm{GeV}$.

We have claimed that with a bound state e.g. with the mass $750 \mathrm{GeV}$ we would get corrected the De Grassi et al. calculation so as to be consistent with exact MPP.

Basically we claim: The leading diagrams treating the bound state as an "elementary particle" (i.e. with formal Feynman rules) though modified by including estimated form factors, we can fit the mass of the bound state so that the diagrams just cancel the instability and make the energy density of the high Higgs field minimum exactly zero, $V_{\text {eff }}\left(\phi_{H} \sim 10^{18} \mathrm{GeV}\right)=0$. That works for a mass $\sim 700$ to $800 \mathrm{GeV}$.

It should be had in mind that, with the experimentally suggested numbers, the Higgs mass squared term in the effective potential for the Higgs field becomes negligible as soon as we go to field scales high above the weak scale. Thus for the interesting scale of the "high field vacuum" the effective potential is practically given by the fourth order self coupling term with coefficient $\lambda_{e f f}\left(\phi_{H}\right)$. Indeed to first approximation one can take the running coupling for the $\lambda$ taken at the value of the Higgs field $\phi_{H}$. If, as we predict[5] by MPP, the effective potential shall just touch zero at the high field vacuum scale, then it means that approximately also the self coupling should just touch zero there.

The running of the Higgs self-coupling is somewhat sensitive to the top-Yukawa coupling $g_{t}(t)$ and thus to the top mass $m_{t}$ from which of course $g_{t}$ is determined experimentally. It is therefore practical to present our prediction of the Higgs mass as a function of the top-mass. One shall then have in mind that our MPP prediction is that the "present vacuum" is just barely stable - it has namely just the same energy density as the "high Higgs field one" - and thus our prediction is that the combination of Higgs and top masses shall be on the line separating the stability and the meta-stability of the present vacuum on the following plot figure 9 .

The Small Instability, Negative Self-coupling at the High field Minimum:

Extrapolated using DeGrassi et al.[64] without our correction, one gets the following value of the running self coupling $\lambda_{\text {run }}\left(10^{18} \mathrm{GeV}\right)$ :

$$
\lambda(\phi \text { "high field" })=-0.01 \pm 0.002 .
$$




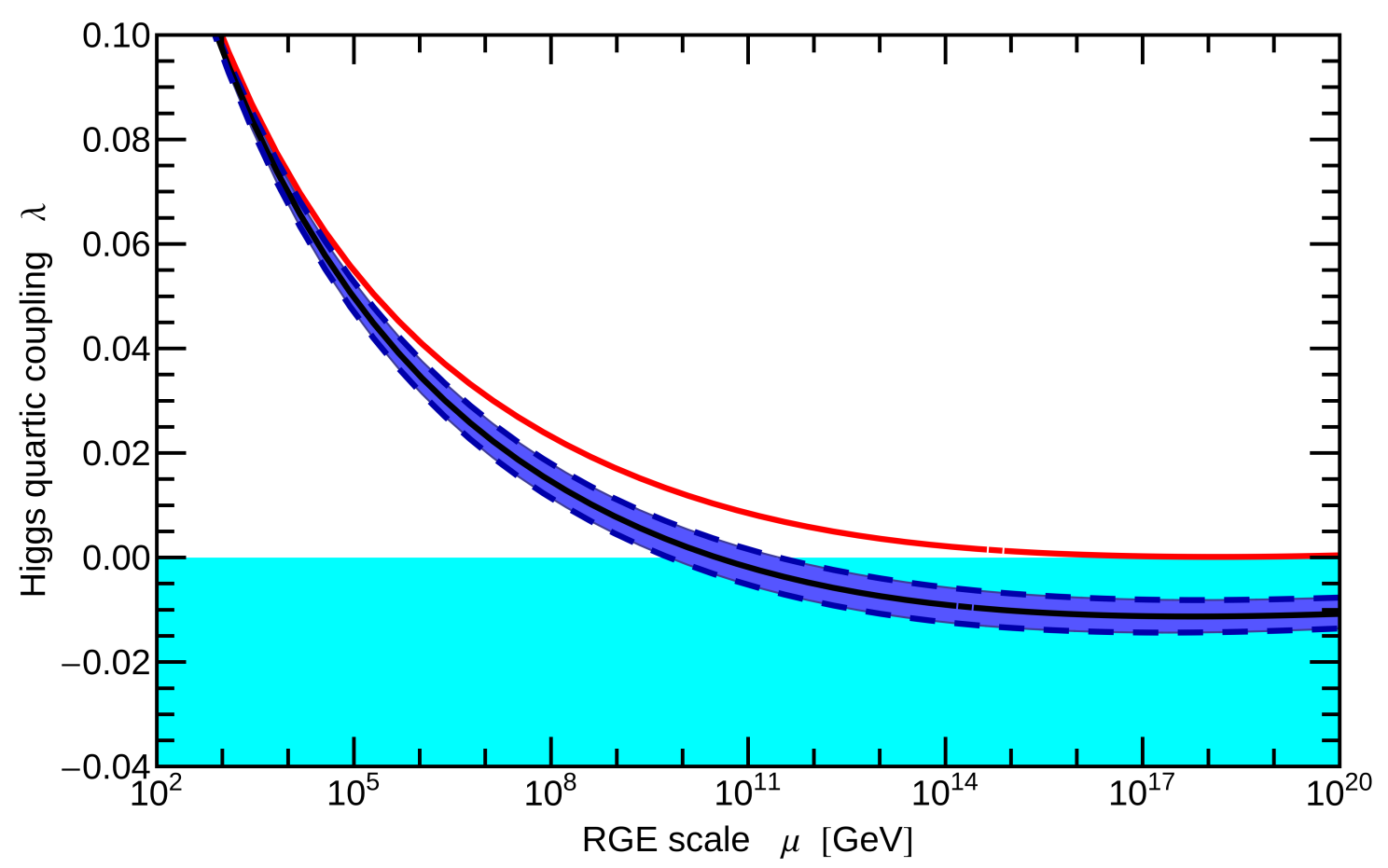

Figure 8: Higgs quartic coupling $\lambda$ RGE running.

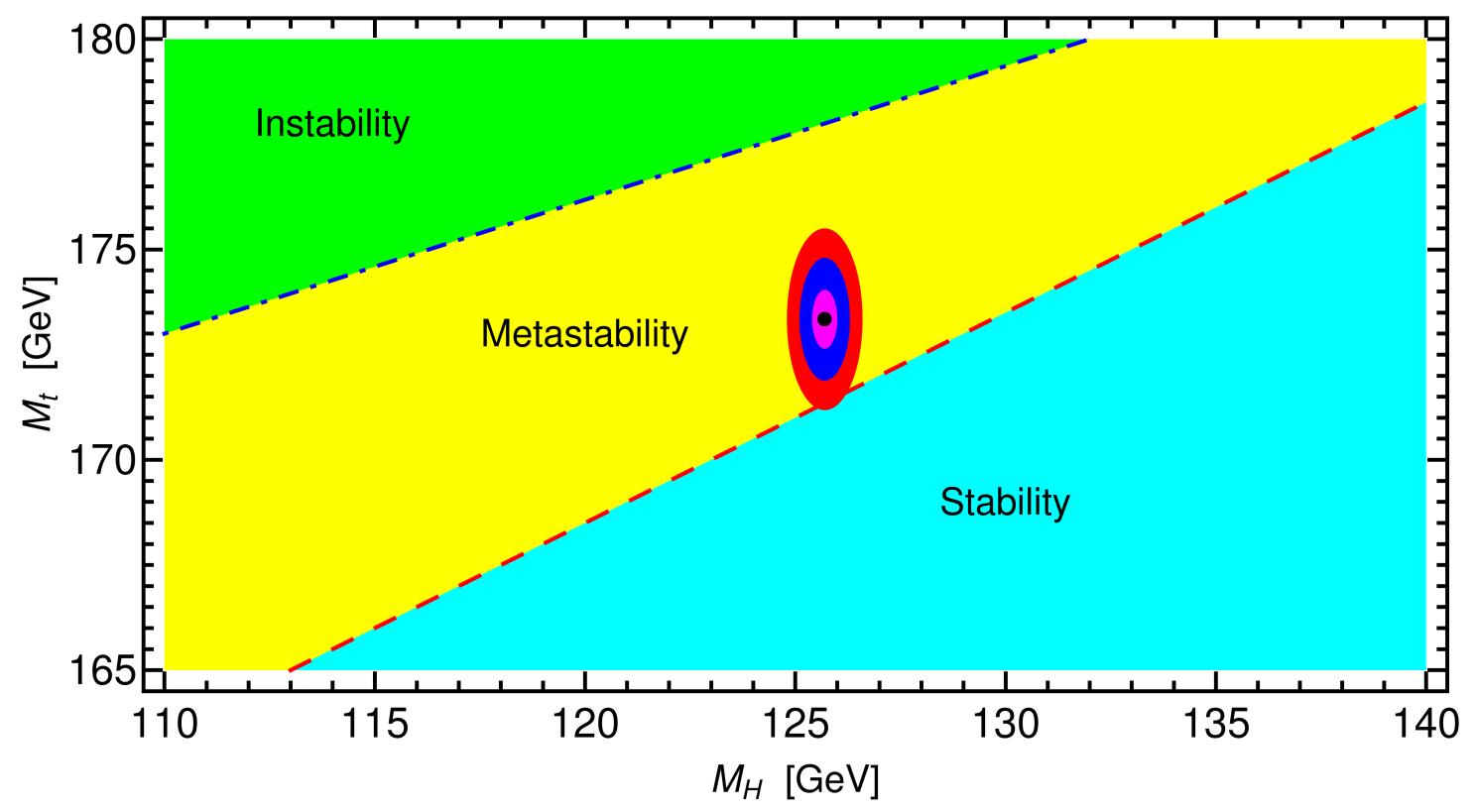

Figure 9: Instability, metastability and stability region. 


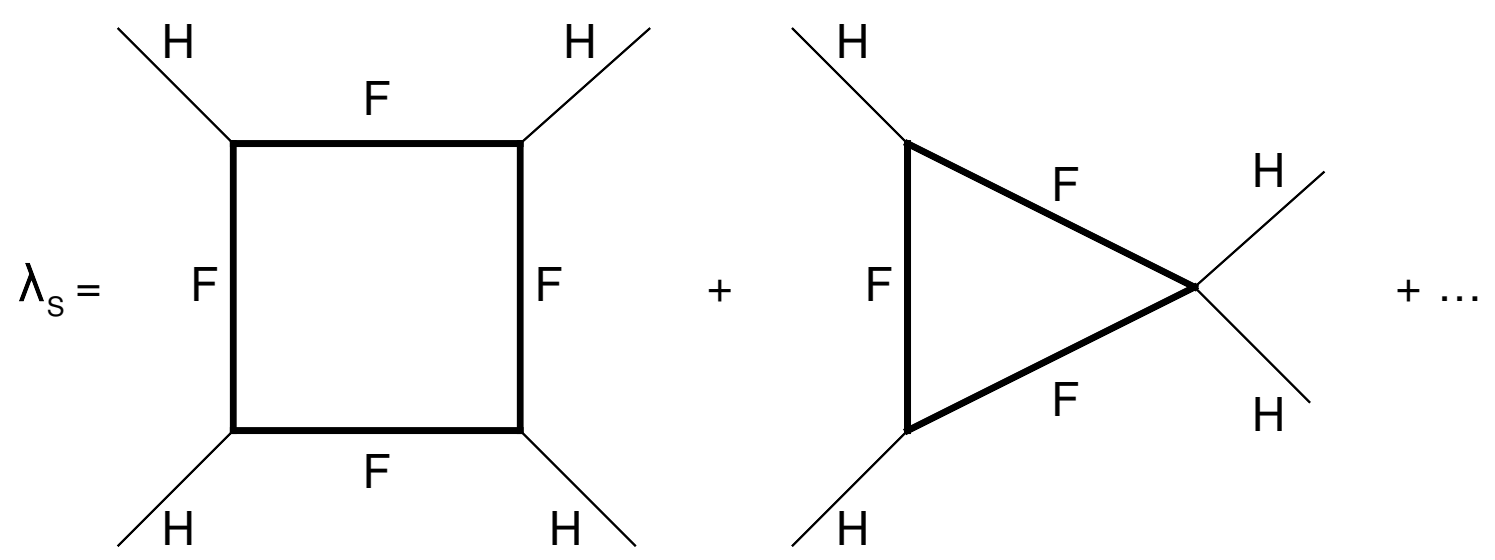

Figure 10: Correction to Higgs-self coupling.

at the high field scale $\phi$ "high field". However, a value very accurately zero is required by the Multiple Point Principle (=MPP).

Since the bound state $\mathrm{F}$ is an extended object we must include a form factor, when using it in Feynman diagrams. A priori it is of course a very doubtful approximation to describe a bound state like ours as if it were a fundamental particle and then only improve the calculation by introducing form factors taking the extended structure of the bound state into account. In the light of the fact that our bound state is expected to be very strongly bound - the mass being about $1 / 3$ of the collective mass of the constituents - there is a better hope that such an as if fundamental particle approximation could be more justified. But it is still one of the very doubtful approximations in our works with this bound state.

Now to obtain form factors you need the radius of the bound state.

Defining a quantity $b$ denoting the radius of the bound state measured with the top quark Compton wave length $1 / m_{t}$ as unit by:

$$
\begin{aligned}
\left\langle\vec{r}^{2}\right\rangle & =3 r_{0}^{2}, \\
r_{0} & =\frac{b}{m_{t}},
\end{aligned}
$$

we obtain a theoretical estimate

$$
b=\sqrt{\frac{\left\langle\vec{r}^{2}\right\rangle}{3}} m_{t} \approx 2.34
$$

Approximating the Bound State as if it were an Elementary Particle, since so Strongly Bound

The dominant diagram/correction - the first (box) diagram on the figure just above - is

$$
\lambda_{S} \approx \frac{1}{\pi^{2}}\left(\frac{6 g_{t}}{b} \times \frac{m_{t}}{m_{S}}\right)^{4}
$$

where we have the estimated or measured values

$$
g_{t}=0.935 ; m_{t}=173 \mathrm{GeV} ; b \approx 2.34
$$


Using the after all rather small deviation from perfect MPP

$$
\lambda_{\text {high field }}=-0.01 \pm 0.002
$$

and requiring it to be cancelled by the correction from the bound state we get the requirement

$$
\lambda_{S}=\frac{1}{\pi^{2}}\left(\frac{6 g_{t}}{b} \times \frac{m_{t}}{m_{F}}\right)^{4} \times(\sim 2) \approx 0.01 \pm 0.002,
$$

where $g_{t}=.935, m_{t}=173 \mathrm{GeV}, b \approx 2.34$ and the factor " $(\sim 2)$ " were taken in to approximate some neglected diagrams.

If a further study should show that the next diagrams add up to roughly as much as the first one, we should include the factor $\sim 2$ to take into account the neglected Feynman diagrams correcting the Higgs self coupling.

The solution w.r.t. the mass of the bound state $m_{F}$ gives

$$
\begin{aligned}
m_{F} & \approx \frac{6 g_{t} m_{t}}{b}\left(\frac{\sim 2}{\pi^{2} \times 0.01 \pm 0.002}\right)^{1 / 4} \\
\approx 2.31 \times 173 \mathrm{GeV} \times 2.1 & =4.9 \times 173 \mathrm{GeV}=850 \mathrm{GeV} \pm 20 \% \\
\text { or without the } \sim 2: & \\
m_{F}=2.31 \times 173 \mathrm{GeV} \times 1.8 & =4.1 \times 173 \mathrm{GeV}=710 \mathrm{GeV} \pm 20 \%
\end{aligned}
$$

\section{Three Agreeing Fits of the Bound State Mass:}

Let us here tell that we soon shall present more bound state calculations than the one just presented for which we give two result values, depending on whether the next term in the Feynman diagram expansion will be important or not. Only the very first - box diagram - term gives what we call "without $\sim 2$ " factor.

The summary, before having presented details of the other crude bound state calculations, is:

$$
\begin{aligned}
m_{F}(\text { from "high field vacuum") } & \approx 850 \mathrm{GeV} \pm 30 \% \text { with } \sim 2 \\
m_{F}(\text { from "high field vacuum") } & \approx 710 \mathrm{GeV} \pm 30 \% \text { without } \sim 2 \\
m_{F} \text { ("condensate vac.") } & \approx 692 \mathrm{GeV} \pm 40 \% \\
m_{F} \text { ("bag estimate") } & \approx 5 m_{t}=865 \mathrm{GeV} \text { (very uncertain). }
\end{aligned}
$$

The agreement of the value " $692 \mathrm{GeV}$ " with the estimate(s) from the completely different vacuum with the high Higgs field " $850 \mathrm{GeV}$ " or " $710 \mathrm{GeV}$ " and with the mass by estimating how strong the top and anti tops can bind $m_{F}$ ("bag estimate") $\approx 865 \mathrm{GeV}$ is encouraging and provides support for our "Multiple Point Principle"!

\section{Condensate}

Fitting Bound State Mass to the "Condensate Vacuum" having Same Energy Density as the "Present" one.[36, 73]

For calculational purposes we approximate the "condensate vacuum" with a crystal (actually it should at least be a fluid, but that may not matter so much for our crude energy density estimate) 
made from the bound states sitting each with 4 neighbors, the tops and anti tops of which are in approximate main quantum number $\mathrm{n}=2$ states seen from the bound state considered.

The MPP-requirement may be written

$$
\begin{aligned}
0 & =m_{F}-\text { "binding per } \mathrm{F}^{\prime \prime} \\
& =m_{F}-\frac{\text { \#neighbors }}{2} \times \text { "binding to neighbor } \mathrm{F}^{\prime \prime} \\
& \approx m_{S}-\frac{4}{2} \times \text { "binding of } \mathrm{F} \text { in } \mathrm{n}=2 \text { arround another } \mathrm{F}^{\prime \prime} \\
& \approx m_{F}-\frac{4}{2} \times \text { "binding of } \mathrm{F}^{\prime \prime} \times \frac{1 / 2^{2}}{1 / 1^{2}} \\
& =m_{F}-\frac{1}{2} \times \text { "binding of } \mathrm{F}^{\prime \prime} \\
& =m_{F}-\frac{1}{2} \times\left(12 m_{t}-m_{F}\right) \\
& =\frac{3}{2} m_{F}-6 m_{t}
\end{aligned}
$$

We here follow an appendix of our earlier work[73] and assume that the structure of the condensate can be approximated as being a diamond lattice structure, so that there are just \#neighbors = 4 neighbors, i.e. other F-particles surrounding each one of them in the lattice. When we count all the binding energy per $\mathrm{F}$ present in the condensate "binding per $\mathrm{F}^{\prime \prime}$ as being the number of neighbors \#neighbors times the binding of one $\mathrm{F}$ to its neighbor "binding to neighbor $\mathrm{F}^{\text {", we double }}$ count, because we count the same binding from both the one $\mathrm{F}$ and from the other one it binds to.

We made then the approximation that the neighboring top quarks and anti top quarks contained in an F neighboring to another one are in effect in the $n=2$ orbit of the latter. Thus we can take the binding energy of a neighboring $\mathrm{F}$ to a given one "binding to neighbor $\mathrm{F}^{\text {" }}$ to be as if the tops and anti tops were in an $n=2$ orbit or some superposition thereof. Thus the binding of the neighbors occur with binding energy "binding of $\mathrm{F}$ in $\mathrm{n}=2$ around another $\mathrm{F}$ ".

As long as we can take the effective Higgs mass for the two lowest orbits $n=1$ and 2 to be zero, we can count that the binding energy, for top say, in the $n=2$ orbit is just one quarter of that in the $n=1$ orbit, provided we can use the same potential of the form $\propto 1 / r$. This result is ensured by an "accidental cancellation" discussed in an earlier article[24], which we now briefly explain.

When one considers a top quark say circulating around a bunch of eleven top or anti top quarks, the attraction is only about half as strong as it would be if these 11 particles were all concentrated in a single point/a center. This is because on the average half of the 11 particles are further away from the center than the considered particle. Thus for such a group of particles binding to each other, one should indeed use an effective coupling strength, say $g_{t}^{2}$, which is only half as strong as what we get formally. In the article[24], in which we calculate the mass of the bound state needed for making the F condensate be degenerate with the present vacuum, we found that this correction by the factor $1 / 2$ in the strength of attraction happens to approximately cancel a couple of other corrections. So that very nicely we could ignore this correction and take it that we had thereby even corrected for the gluon exchange attraction, as well as for the effect of exchange of W's and Z's (or what we call eaten Higgses, since it is mainly the longitudinal $\mathrm{Z}$ or $\mathrm{W}$ that matters). 
In this way the calculation simplifies appreciably and even for an F-particle, which consists of tops and anti tops, the ratio of the binding energies in the two lowest orbits should be $1 / 2^{2}=1 / 4$.

From the last step in (7.7) we easily derive of course that

$$
m_{F}=\frac{2}{3} \times 6 m_{t}=4 m_{t}=4 \times 173 \mathrm{GeV}=692 \mathrm{GeV} \text { agreeing well with } 710 \mathrm{GeV} \text { or } 850 \mathrm{GeV} \text { ! }
$$

\section{Bag}

\section{Estimating How 6 Top + 6 Anti top Bind}

We have made an estimate of the mass of the bound state of 6 top +6 anti top quarks, to be crudely

$$
m_{\text {bound state }}=875 \mathrm{GeV} \text { or } 792 \mathrm{GeV} \pm \text { say } 40 \% .
$$

Our method mainly used a bag-model estimation, in which the bag meant a region where the Higgs field was reduced to $\sim 0$.

Such a region, where the Higgs field expectation value goes approximately to zero, we expect to occur for sufficiently strong interaction of the top and anti top quarks with the Higgs.

Assuming that such a bag of very small Higgs field value becomes significant we estimated, using some further adjustments of how much the top or anti top quarks may tunnel out of the bag, how to obtain the minimal energy ansatz for the bag system with the 12 top or anti tops inside. We managed to get the mass of the bound state down to $\sim 7 m_{t} \approx 1216 \mathrm{GeV}$. Then, however, including further corrections such as the exchange of W's and Z's (or, as we call them, eaten Higgses) and gluons, crude corrections of the bag calculation rather give

$$
M_{\text {corrected eaten }+ \text { gluons }}=5.06 m_{t}=875 \mathrm{GeV} \text {. }
$$

\section{Hierarchy}

\section{"Solve" Hierarchy problem[21, 22]}

Having made a fine-tuning theory/model/rule we have at least the chance to have our finetuning theory MPP give the experimentally observed order of magnitude for the Higgs mass say. And indeed we predict the right order for the logarithm of the scale range over which $\mu$ has to run to get the running top-yukawa-coupling $g_{\text {t run }}(\mu)$ go from 0.4 at the $10^{18} \mathrm{GeV}$ to the 0.935 needed at the weak scale, from the requirement of the "condensate vacuum" being degenerate with the "present one".

It should be stressed that, on the figure illustrating the running top Yukawa coupling $g_{t}(t)$ as a function of the scale(parameter) $t$, our multiple point principle predicts the value of this top Yukawa both at about the Planck scale $10^{18} \mathrm{GeV}$ and at the weak scale. We can thus in principle use the knowledge of the rate of running, the beta-function, to predict from MPP the scale of the weak interactions. Our success is that this weak scale becomes very much smaller than the Planck scale, and in fact it even comes out very well! In that sense we did not really "solve" the hierarchy problem, because it is still a fine tuning, but we have by MPP provided a theory for the fine tuning! 


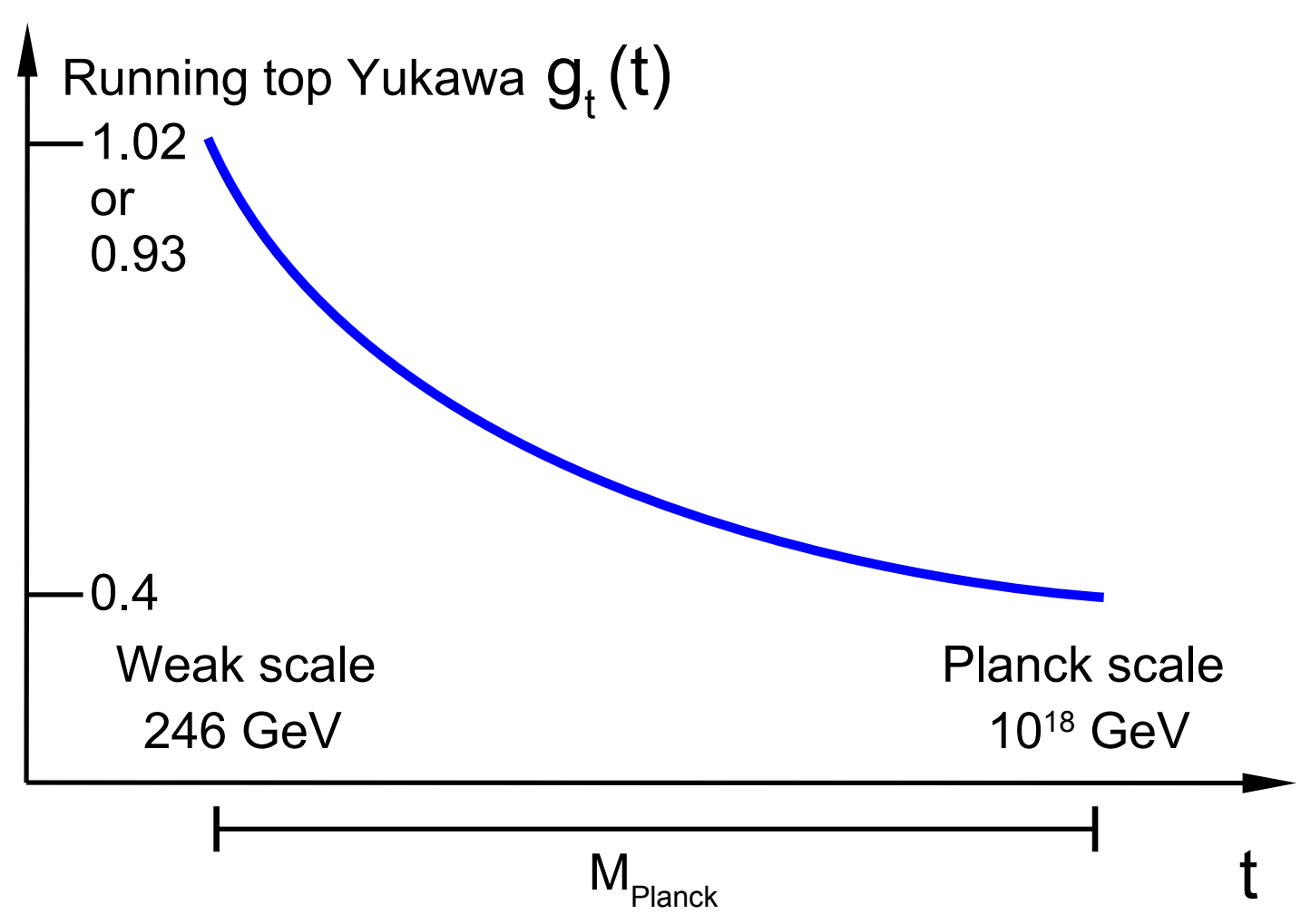

Figure 11: Top Yukawa coupling RGE running.

\section{Conclusion}

\section{Conclusion}

The remarkable coincidence, that our three mass estimations coincide provides evidence in favour of the truth of our model, with the Multiple Point Principle and the bound state!

Some Achievements of our Model MPP and Strongly Bound State of 6 Top + 6 Anti top quarks (in pure Standard Model)

I must mention the following achievements most of which I did not have much time for:

- Hierarchy problem: The fine-tuning caused by our MPP requirements combined with the assumption, that the Higgs field in the "high field vacuum" is of the order of the Planck scale (or only a bit under) leads to the scale problem being solved, in the sense that the Higgs mass and weak scale get fixed to be exponentially much lower than the Planck scale, and that in fact we get very close to the right size for the logarithm.

- $g_{t}$ : Froggatt and I estimated the value of the top-Yukawa-coupling $g_{t}$ needed for MPP, in the sense that it represents a phase transition value between the "condensate vacuum" and the "present vacuum". We found the phase transition value $g_{t}$ phase transition $=1.02 \pm 14 \%$, agreeing with experiment $g_{t \exp }=0.93_{5}$.

- Stability: Explaining that the Higgs mass just puts our vacuum on the borderline of being meta-stable[5]. 
- Correction to Stability: ...even very accurately, if we take seriously the very small correction due to the bound state by Laperashvili, Das, and myself.

- The "condensate vacuum" can be used for a model for dark matter as pearl size balls of the "condensate vacuum" surrounded necessarily by a skin - the transition surface - that is then pumped up by ordinary matter, carbon say, to a pressure of the order of that in a white dwarf star. Such pearls may be useful for

- Dark matter [73, 74, 75].

- making supernovae explode so as to throw sufficient material out so that we can observe them [76].

- Explaining the two bursts of neutrinos observed with $\sim 5$ hours time difference in SN1989A in the Big Magellanic Cloud [76].

- helps r-process fit?

- explain ratio of dark to normal matter being of order $6[74,75]$.

\section{Achievements of MPP in Extended Models (i.e. not only Standard Model):}

- Value of Cosmological Constant: With Roman Nevzorov we got values for the CC using the "same version" of MPP and an almost supersymmetric vacuum state.

- Number of families : Prior to having formulated MPP we fitted fine structure constants in an extension of the Standard Model "AntiGUT" in which each family of fermions has its own set of gauge bosons. We - including Brene and Don Bennett and me - predicted the number of families, which was not known yet at that time.

\section{Encouragement for Theoreticians to Calculate More Accurately This Bound State}

At the end I would stress:

Since our picture is PURE STANDARD MODEL, everything can in principle be CALCULATED!

So it is only a question of better techniques - Bethe Salpeter Equation ?[77] - or better computers and use of them - lattice theory with Higgs field on the lattice ? - to obtain more solid and accurate checks of MPP and calculation of the bound state mass than my crude estimates.

And this is just a work for the theoreticians (among the students say).

Then there should pop up some peaks - like the joke of Pich's (mentioned at this conference as a joke[78] from CMS) - by themselves, when the experimentalists make the plots.

If not it would mean that the Standard Model were not right also nonperturbatively, if our calculations were right.

\section{Acknowledgement}

Holger Bech Nielsen thanks the Niels Bohr Institute for allowance to stay as emeritus and for some travel support, which though seems to have run out before the travel to Corfu and back were finished. 


\section{References}

[1] D.L. Bennett and H.B. Nielsen, Int. J. Mod. Phys. A9, 5155 (1994).

[2] D.L. Bennett and H.B. Nielsen, Int. J. Mod. Phys. A14, 3313 (1999).

[3] D.L. Bennett, C.D. Froggatt and H.B. Nielsen, in Proc. 27th Int. Conf. on High En- ergy Physics, Glasgow, Scotland, 1994, eds. P. Bussey and I. Knowles (IOP Publishing Ltd., 1995), p. 557.

[4] D.L. Bennett, C.D. Froggatt and H.B. Nielsen, in Perspectives in Particle Physics ÂŠ94, eds. D. Klabu.car, I. Picek and D. Tadi.c (World Scientific, Singapore, 1995), p. 255.

[5] C.D. Froggatt and H.B. Nielsen, Phys. Lett. B368, 96 (1996).

[6] D. L. Bennett, PhD thesis at The Niels Bohr Institute, Copenhagen,"Multiple Point criticallity. Fine tuning and Fundamental Physics: Prediction for Gauge Couplings gives $\alpha^{-1}=136.8 \pm 9 . " 31$. May, 1996.

[7] L.V. Laperashvili, Yad. Fiz. 57, 501 (1994) [Phys. Atom. Nucl. 57, 471 (1994)].

[8] C.D. Froggatt, L.V. Laperashvili, R.B. Nevzorov and H.B. Nielsen, Phys. Atom. Nucl. 67, 582 (2004) [Yad. Fiz. 67, 601 (2004)], arXiv:hep-ph/0310127.

[9] C.D. Froggatt, L.V. Laperashvili and H.B. Nielsen, Phys. Atom. Nucl. 69, 67 (2006), hep-ph/0407102.

[10] D.L. Bennett, L.V. Laperashvili and H.B. Nielsen, Relation between fine structure constants at the Planck scale from multiple point principle, in Proc. 9th Workshop: What Comes Beyond the Standard Models, Bled, Slovenia, eds. M. Breskvar et al. (DMFA, Zaloznistvo, Ljubljana, 2006), p. 10, arXiv:hep-ph/0612250.

[11] D.L. Bennett, L.V. Laperashvili and H.B. Nielsen, Finestructure constants at the Planck scale from multiple point principle, in Proc. 10th Workshop on What Comes Beyond the Standard Model, Bled, Slovenia, 17ÂÛ́27 Jul 2007, arXiv:0711.4681.

[12] C.D. Froggatt, R.B. Nevzorov and H.B. Nielsen, Smallness of the cosmological constant and the multiple point principle, J. Phys. Conf. Ser. 110, 072012 (2008), arXiv:0708.2907.

[13] C.D. Froggatt, R.B. Nevzorov, H.B. Nielsen, A.W. Thomas, Phys. Lett. B737, 167 (2014), arXiv:1403.1001.

[14] C.D. Froggatt, R.B. Nevzorov, H.B. Nielsen, A.W. Thomas, On the smallness of the cosmological constant in SUGRA models with Planck scale SUSY breaking and degenerate vacua, 2015 European Physical Society Conference on High Energy Physics (EPS-HEP 2015), 22-29 Jul, 2015, Vienna, Austria; arXiv:1510.05379.

[15] Yuta Hamada, Hikaru Kawai, and Kiyoharu Kawana, Prog. Theor. Exp. Phys. (2015) 123B03 doi: 10.1093/ptep/ptv168

[16] Hamada Y., Kawai H., and Oda K. y. , Phys. Rev. D 87, 053009 (2013); 89, 059901 (2014) [erratum] [hep-ph]] [Search inSPIRE]. (doi:10.1103/PhysRevD.89.059901)

[17] Kiyoharu Kawana, Prog. Theor. Exp. Phys. (2015) 023B04 doi: 10.1093/ptep/ptv006

[18] Yuta Hamada, Hikaru Kawai, and Kiyoharu Kawana, Int. J. Mod. Phys. A 29, 1450099 (2014)

[19] Naoyuki Haba, Hiroyuki Ishida, Nobuchika Okada, Yuya Yamaguchi, Prog Theor Exp Phys (2017) 2017 (1): 013B03. DOI: https://doi.org/10.1093/ptep/ptw186 "Multiple-point principle with a scalar singlet extension of the standard model" 
[20] Hamada, Kinya Oda, ... wwwhep.s.kanazawa-u.ac.jp/SI2014/slides/Oda.pdf

[21] C.D. Froggatt and H.B. Nielsen, Trying to understand the Standard Model parameters. Invited talk by H.B. Nielsen at the "XXXI ITEP Winter School of Physics", Moscow, Russia, 18-26 February, 2003; Surveys High Energy Phys. 18, 55-75 (2003); hep-ph/0308144.

[22] C.D. Froggatt, H.B. Nielsen and L.V. Laperashvili, Hierarchy-problem and a bound state of 6 t and 6 anti-t, in: Proceedings of Coral Gables Conference on Launching of Belle Epoque in High-Energy Physics and Cosmology (CG 2003), Ft. Lauderdale, Florida, 17-21 December, 2003.

[23] C.D. Froggatt, H.B. Nielsen and L.V. Laperashvili, Int. J. Mod. Phys. A 20, 1268 (2005); hep-ph/0406110.

[24] C.D. Froggatt and H.B. Nielsen, Phys. Rev. D 80, 034033 (2009); arXiv:0811.2089.

[25] C.D. Froggatt, H.B. Nielsen, Hierarchy Problem and a New Bound State, in Proc. to the Euroconference on Symmetries Beyond the Standard Model, p.73, Slovenia, Portoroz, 2003 (DMFA, Zaloznistvo, 2003); ArXiv: hep-ph/0312218.

[26] C.D. Froggatt, The Hierarchy problem and an exotic bound state, in: Proceedings of 10th International Symposium on Particles, Strings and Cosmology, (PASCOS 04), Boston, Massachusetts, 16-22 Aug, 2004. Published in: "Boston 2004, Particles, strings and cosmology", pp.325-334; hep-ph/0412337.

[27] C.D. Froggatt, L.V. Laperashvili and H.B. Nielsen, A New bound state $6 t+6$ anti-t and the fundamental-weak scale hierarchy in the Standard Model, in: Proceedings of 13th International Seminar on High-Energy Physics: QUARKS-2004, Pushkinskie Gory, Russia, 24-30 May, 2004; hep-ph/0410243.

[28] C.D. Froggatt, L.V. Laperashvili and H.B. Nielsen, Phys. Atom. Nucl. 69, 67 (2006) [Yad. Fiz. 69, 3 (2006)]; hep-ph/0407102.

[29] C.D. Froggatt, L.V. Laperashvili, R.B. Nevzorov and H.B. Nielsen, The Production of $6 t+6 \bar{t}$ bound state at colliders. A talk given by H.B. Nielsen at CERN, 2008, preprint CERN-PH-TH/2008-051.

[30] C.D. Froggatt, L.V. Laperashvili, R.B. Nevzorov, H.B. Nielsen and C.R. Das, New Bound States of Top-anti-Top Quarks and T-balls Production at Colliders (Tevatron, LHC, etc.), Report CHEP-PKU-1-04-2008, CERN (2008); arXiv:0804.4506.

[31] C.D. Froggatt and H.B. Nielsen, in: Proceedings to the 34th International Conference on High Energy Physics (ICHEP 2008), 30 Jul - 5 Aug 2008, Philadelphia, Pennsylvania; arXiv:0810.0475.

[32] C.R. Das, C.D. Froggatt, L.V. Laperashvili and H.B. Nielsen, Int. J. Mod. Phys. A 26, 2503 (2011); arXiv:0812.0828.

[33] C.D. Froggatt, C.R. Das, L.V. Laperashvili and H.B. Nielsen, New indications of the existence of the 6 top-anti-top quark bound states in the LHC experiments, a talk by L.V. Laperashvili at the Conference "Quarkonium-2012", Moscow, Russia, MEPhI, November, 12 - 16, 2012, arXiv:1212.2168 [hep-ph]; Yad. Fiz., 76, 172 (2013).

[34] C.D. Froggatt, C.R. Das, L.V. Laperashvili, H.B. Nielsen, Int. J. Mod. Phys. A30, 1550132 (2015); arXiv: 1501.00139.

[35] L. V. Laperashvili, H. B. Nielsen and C. R. Das, "New results at LHC confirming the vacuum stability and Multiple Point Principle,” Int. J. Mod. Phys. A 31 (2016) no.08, 1650029 doi:10.1142/S0217751X16500299 [arXiv:1601.03231 [hep-ph]]. 
[36] Holger Frits Bech Nielsen "Simple Mass-estimates for New LHC-Resonances F(750) and "1.8 TeV" as 6 Top plus 6 Anti top Bound states and Combinations thereof”, arXiv:1607.07907.

[37] ATLAS Collaboration, JHEP 09 (2016) 001; arXiv:1606.03833.

[38] CMS Collaboration, Phys. Rev. Lett. 117, 051802 (2016) ; arXiv:1606.04093.

[39] ATLAS Collaboration, ATLAS-CONF-2016-059, "Search for scalar diphoton resonance with 15.4 $\mathrm{fb}^{-1}$ of data collected at $\sqrt{s}=13 \mathrm{TeV}$ in 2015 and 2016 with the ATLAS detector"

[40] CMS-Collaboration, CMS-PAS-EXO-16-027, "Search for resonant production of high mass photon pairs using $12.9 \mathrm{fb}^{-1}$ of proton-proton collisions at $\sqrt{s}=13 \mathrm{TeV}$ and combined interpretation of searches at 8 and $13 \mathrm{TeV}$ "

[41] C. D. Froggatt and H. B. Nielsen, "Production and Decay of 750 Gev state of 6 top and 6 anti top quarks," arXiv:1605.03909 [hep-ph].

[42] "Dark Energy Density in SUGRA models and degenerate vacua" C.D. Froggatt, H.B. Nielsen, R. Nevzorov, A.W. Thomas. Apr 27, 2017. ADP-17-18-T1024 arXiv:1704.08453 [hep-ph]

[43] Handbook of LHC Higgs Cross Sections: 4. "Deciphering the Nature of the Higgs Sector LHC Higgs Cross Section Working Group” (D. de Florian (ITeDA, Buenos Aires) et al.). Oct 25, 2016. 868 pp. FERMILAB-FN-1025-T, arXiv:1610.07922 [hep-ph]

[44] "On the smallness of the cosmological constant" C.D. Froggatt (Glasgow U.), R. Nevzorov (Adelaide U. \& Adelaide U., Sch. Chem. Phys. \& Moscow, ITEP), H.B. Nielsen (Bohr Inst.), A.W. Thomas (Adelaide U. \& Adelaide U., Sch. Chem. Phys.). Oct 24, 2014. 6 pp. Published in Nucl.Part.Phys.Proc. 273-275 (2016) 1465-1470 ADP-14-31-T890 DOI:

10.1016/j.nuclphysbps.2015.09.237 Conference: C14-07-02 Proceedings, arXiv:1410.6620 [hep-ph]

[45] "On the smallness of the dark energy density in split SUSY models inspired by degenerate vacua", C.D. Froggatt (Glasgow U.), R. Nevzorov (U. Hawaii, Honolulu), H.B. Nielsen (Bohr Inst.). 2013. 3 pp. Published in AIP Conf.Proc. 1560 (2013) 300-302 DOI: 10.1063/1.4826779 Conference: C12-05-29 Proceedings

[46] "Dark Energy density in models with Split Supersymmetry and degenerate vacua" C. Froggatt (Glasgow U.), R. Nevzorov (Hawaii U.), H.B. Nielsen (Bohr Inst.). Mar 2011. 20 pp. Published in Int.J.Mod.Phys. A27 (2012) 1250063 UH511-1164-2011 DOI: 10.1142/S0217751X12500637, arXiv:1103.2146 [hep-ph]

[47] "Dark Energy density in Split SUSY models inspired by degenerate vacua" Colin Froggatt (Glasgow U.), Roman Nevzorov (Hawaii U.), Holger Bech Nielsen (Bohr Inst.). Dec 2010. 4 pp. Published in PoS ICHEP2010 (2010) 442 Prepared for Conference: C10-07-21 Proceedings arXiv:1012.5121 [hep-ph]

[48] "On the Smallness of the Cosmological Constant in SUGRA Models Inspired by Degenerate Vacua" C.D. Froggatt, R. Nevzorov (Glasgow U.), H.B. Nielsen (Bohr Inst.). Sep 2009. 5 pp. Published in AIP Conf.Proc. 1200 (2010) 1093-1096 DOI: 10.1063/1.3327546 Talk given at Conference: C09-06-05 Proceedings, arXiv:0909.4703 [hep-ph]

[49] “Cosmological Constant in SUGRA Models Inspired by Degenerate Vacua” C.D. Froggatt, R. Nevzorov (Glasgow U.), H.B. Nielsen (Bohr Inst.). Oct 2008. 4 pp. Talk given at Conference: C08-07-30 Proceedings, arXiv:0810.0524 [hep-th] 
[50] "On the origin of approximate custodial symmetry in the Two-Higgs Doublet Model” C.D. Froggatt (Glasgow U. \& Bohr Inst.), R. Nevzorov (Glasgow U.), H.B. Nielsen (Bohr Inst.), D. Thompson (Glasgow U.). Jun 2008. 64 pp. Published in Int.J.Mod.Phys. A24 (2009) 5587-5637 CERN-PH-TH-2008-058 DOI: 10.1142/S0217751X09047442, arXiv:0806.3190 [hep-ph]

[51] "New Bound States of Top-anti-Top Quarks and TấL ̌̌ balls Production at Colliders (Tevatron, LHC, etc.)" C.D. Froggatt (Glasgow U.), L.V. Laperashvili (Moscow, ITEP), R.B Nevzorov (Glasgow U. \& Moscow, ITEP), H.B. Nielsen (Bohr Inst. \& CERN), C.R. Das (Peking U.). Apr 2008. 43 pp. CHEP-PKU-1-04-2008, arXiv:0804.4506 [hep-ph]

[52] "Multiple point principle as a mechanism for the suppression of FCNC and CP-violation phenomena in the 2HDM" C.D. Froggatt, R. Nevzorov (Glasgow U.), H.B. Nielsen (Bohr Inst.). Oct 2007. 4 pp. Published in In *Karlsruhe 2007, SUSY 2007* 710-713 To appear in the proceedings of Conference: C07-07-26, p.710-713 Proceedings, arXiv:0710.2457 [hep-ph]

[53] "Enhanced Higgs boson production and avoidance of CP-violation and FCNC in the MPP inspired 2HDM" C.D. Froggatt, R. Nevzorov (Glasgow U.), H.B. Nielsen (Bohr Inst.). Aug 2007. 3 pp. Published in J.Phys.Conf.Ser. 110 (2008) 062010 DOI: 10.1088/1742-6596/110/6/062010 Talk given at Conference: C07-07-19 Proceedings, arXiv:0708.2905 [hep-ph]

[54] "Fixed point scenario in the Two Higgs Doublet Model inspired by degenerate vacua" C.D. Froggatt, R. Nevzorov (Glasgow U.), H.B. Nielsen (Bohr Inst.), D. Thompson (Glasgow U.). Aug 2007. 16 pp. Published in Phys.Lett. B657 (2007) 95-102 DOI: 10.1016/j.physletb.2007.10.010, arXiv:0708.2903 [hep-ph]

[55] "Implementation of the multiple point principle in the two-Higgs doublet model of type II" Colin D. Froggatt (Glasgow U.), Larisa Laperashvili (IMSc, Chennai), Roman Nevzorov (Southampton U.), Holger Bech Nielsen (Bohr Inst.), Marc Sher (William-Mary Coll.). Feb 2006. 26 pp. Published in Phys.Rev. D73 (2006) 095005 DOI: 10.1103/PhysRevD.73.095005, hep-ph/0602054

[56] "On the smallness of the cosmological constant in SUGRA models" C. Froggatt (Glasgow U.), R. Nevzorov (Southampton U.), H.B. Nielsen (Bohr Inst.). Nov 2005. 28 pp. Published in Nucl.Phys. B743 (2006) 133-152 DOI: 10.1016/j.nuclphysb.2006.03.008, hep-ph/0511259

[57] “The Two Higgs doublet model and the multiple point principle” C.D. Froggatt (Glasgow U.), L.V. Laperashvili (Moscow, ITEP), R.B. Nevzorov (Southampton U. \& Moscow, ITEP), H.B. Nielsen (Bohr Inst.), M. Sher (William-Mary Coll.). Dec 2004. 12 pp. Published in Bled Workshops Phys. 5 (2004) no.2, 28-39 GUTPA-04-12-01 Conference: C04-07-19.9 Proceedings, hep-ph/0412333

[58] "No-scale supergravity and the multiple point principle" C. Froggatt (Glasgow U.), L. Laperashvili (Moscow, ITEP), R. Nevzorov (Southampton U. \& Moscow, ITEP), H.B. Nielsen (Bohr Inst.). Dec 2004. 11 pp. Published in Bled Workshops Phys. 5 (2004) no.2, 17-27 Conference: C04-07-19.9 Proceedings, hep-ph/0411273

[59] "Cosmological constant in SUGRA models and the multiple point principle" C. Froggatt (Glasgow U.), L. Laperashvili, R. Nevzorov (Moscow, ITEP), H.B. Nielsen (Bohr Inst.). Oct 2003. 18 pp. Published in Phys.Atom.Nucl. 67 (2004) 582-589, Yad.Fiz. 67 (2004) 601-608 DOI: 10.1134/1.1690068 Talk given at Conference: C02-12-02.1 Proceedings, hep-ph/0310127

[60] D. Bennett, H.B. Nielsen, I Picek Phys.Lett 208B, 275 (1988)

[61] D. Bennett, N. Brene, H.B. Nielsen in recent developments of Quantum Field Theory, Proceedings of the Niels Bohr Centeneal Conference, Copenhagen 1985,eds. Ambjørn, Durhuus, Petersen, North Holland, Amsterdam 1985. 
[62] H. B. Nielsen, Acta Physica Polonica Vol B20,(1989), "Random Dynamics... Fine structure constants."

[63] D. L. Bennett, H. B. Nielsen and C. D. Froggatt, "Standard model parameters from the multiple point principle and anti-GUT," In *Seoul 1997, Recent developments in nonperturbative quantum field theory* 362-393 [hep-ph/9710407].

[64] G. Degrassi, S. Di Vita, J. Elias-Miro, J.R. Espinosa, G.F. Giudice, G. Isidori and A. Strumia, JHEP 1208, 098 (2012); arXiv:1205.6497.

[65] D. Buttazzo, G. Degrassi, P.P. Giardino, G.F. Giudice, F. Salab, A. Salvio, A. Strumia, JHEP 1312, 089 (2013); arXiv:1307.3536.

[66] C. D. Froggatt, H. B. Nielsen and Y. Takanishi, "Standard model Higgs boson mass from borderline metastability of the vacuum,” Phys. Rev. D 64 (2001) 113014 doi:10.1103/PhysRevD.64.113014 [hep-ph/0104161].

[67] Graham Ross, www.ep.ph.bham.ac.uk/general/seminars/.../Graham-Ross-2013.pdf; Andreas Birkedal, Zackaria Chacko, and Mary K. Gaillard, Journal of High Energy Physics "Little supersymmetry and the supersymmetric little hierarchy problem"; Riccardo Barbieri, Alessandro Strumia,. "The LEP paradox". arXiv:hep-ph/0007265

[68] H. B. Nielsen and C. D. Froggatt, "Influence from the future" Proceedings of the 5th Hellenic School and Workshops on Elementary Particle Physics; Corfu 1995, Conference C95-09-051, [arXiv:hep-ph/9607575].

[69] Steven Weinberg “The cosmological constant problem” Rev. Mod. Phys. 61, 1 (1989)

[70] MosterÃøn J., (2005), Anthropic Explanations in Cosmology, in Hajek, ValdÃl's and Westerstahl (eds.), Proceedings of the 12th International Congress of Logic, Methodology and Philosophy of Science; http://philsci-archive.pitt.edu/1658/"

[71] H. B. Nielsen and M. Ninomiya, "Degenerate vacua from unification of second law of thermodynamics with other laws," Bled Workshops Phys. 12 (2011) no.2, 199 [hep-th/0701018].

[72] Nicolai Stillits, Cand. Scient. thesis at the Niels Bohr Institute “ "Darwinistic " Inspired High Energy Physics: Random dynamics, Mutiple Point Criticallity Principle, Non Locality and Supersymmetry"

[73] C. D. Froggatt and H. B. Nielsen, “Tunguska Dark Matter Ball,” Int. J. Mod. Phys. A 30 (2015) no.13, 1550066 doi:10.1142/S0217751X15500669 [arXiv:1403.7177 [hep-ph]].

[74] C. D. Froggatt and H. B. Nielsen, Phys. Rev. Lett. 95 (2005) 231301 [arXiv:astro-ph/0508513]

[75] C.D. Froggatt and H.B. Nielsen, "Dark matter from encapsulated atoms," Bled Workshops Phys. 6 (2005) no.2, 32-39 Prepared for Conference: C05-07-19.3 Proceedings [arXiv:astro-ph/0512454]

[76] C. D. Froggatt and H. B. Nielsen, Mod.Phys.Lett. A30 (2015) no.36, 1550195 arXiv:1503.01089 [astro-ph.HE]

[77] H. Bethe, E. Salpeter (1951). "A Relativistic Equation for Bound-State Problems". Physical Review 84 (6): 1232. Bibcode:1951PhRv...84.1232S. doi:10.1103/PhysRev.84.1232.

[78] CMS Collaboration, CMS PAS B2G-16-010, "Search for diboson resonances in the semi-leptonic X $\rightarrow \mathrm{ZV} \rightarrow l^{+} l^{-} q \bar{q}$ final states at $\sqrt{s}=13 \mathrm{TeV}$ with CMS" 